\title{
Resonant scattering of X-ray emission lines in the hot intergalactic medium
}

\author{
Eugene Churazov • Irina Zhuravleva . \\ Sergey Sazonov • Rashid Sunyaev.
}

Received: date / Accepted: date

\begin{abstract}
While very often a hot intergalactic medium (IGM) is optically thin to continuum radiation, the optical depth in resonant lines can be of order unity or larger. Resonant scattering in the brightest X-ray emission lines can cause distortions in the surface brightness distribution, spurious variations in the abundance of heavy elements, changes in line spectral shapes and even polarization of line emission. The magnitude of these effects not only depends on the density, temperature and ionization state of the gas, but is also sensitive to the characteristics of the gas velocity field. This opens a possibility to use resonant scattering as a convenient and powerful tool to study IGM properties. We discuss the application of these effects to galaxy clusters.
\end{abstract}

Keywords X-rays · clusters of galaxies · radiative transfer

\section{Introduction}

Hot $\left(10^{6}-10^{8} \mathrm{~K}\right) \mathrm{X}$-ray emitting astrophysical plasmas are often optically thin with respect to free-free absorption. One famous example is the coronae of the Sun and other stars. It was realized early (e.g. Elwert 1956) that in the solar corona the optical depth in certain resonant lines of oxygen, neon and iron can be larger than unity and a

This work was supported by the DFG grant CH389/3-2, grant NSh-5069.2010.2, grant RFFI 09-02-00867 and by programs P-04 and OFN-16 of the Russian Academy of Sciences. IZ thanks the International Max Planck Research School (IMPRS) in Garching. SS acknowledges the support of the Dynasty Foundation.

E. Churazov

Max Planck Institute for Astrophysics, Karl-Schwarzschild str. 1, Garching, 85741, Germany and Space Research Institute, Profsoyuznaya str. 84/32, Moscow, 117997, Russia

I. Zhuravleva

Max Planck Institute for Astrophysics, Karl-Schwarzschild str. 1, Garching, 85741, Germany

Sergey Sazonov

Space Research Institute, Profsoyuznaya str. 84/32, Moscow, 117997, Russia

Rashid Sunyaev

Max Planck Institute for Astrophysics, Karl-Schwarzschild str. 1, Garching, 85741, Germany and Space Research Institute, Profsoyuznaya str. 84/32, Moscow, 117997, Russia 
radiative transfer problem has to be solved in order to get the correct line ratios (e.g. Acton 1978; Rugge \& McKenzie 1985).

Below we concentrate on the resonant scattering in the hot plasma of galaxy clusters. Hot plasma is the dominant constituent of baryons in clusters, making $10-15 \%$ of the total cluster mass of $10^{14}-10^{15} M_{\odot}$. Bremsstrahlung, recombination continuum and emission lines excited by electron collisions are the main emission components in this plasma. In spite of such a large gas mass, the optical depth of the hot plasma for free-free absorption is extremely low in the X-ray regime. This is immediately clear from a comparison of the observed X-ray luminosity of the brightest clusters, $L_{X} \sim 10^{45} \mathrm{erg} \mathrm{s}^{-1}$, with the black body radiation of a Mpc size object with a temperature $T \sim 10^{7}-10^{8} \mathrm{~K}$ :

$$
\tau_{f f} \approx \frac{L_{X}}{4 \pi R^{2} \sigma T^{4}}=1.5 \cdot 10^{-33}\left(\frac{L_{X}}{10^{45} \mathrm{erg} \mathrm{s}^{-1}}\right)\left(\frac{R}{\mathrm{Mpc}}\right)^{-2}\left(\frac{T}{10^{8} \mathrm{~K}}\right)^{-4},
$$

where $R$ is the characteristic cluster size. The depth for Thomson scattering is also low:

$$
\tau_{T}=\sigma_{T} n_{e} R=2 \cdot 10^{-3}\left(\frac{n_{e}}{10^{-3} \mathrm{~cm}^{-3}}\right)\left(\frac{R}{\mathrm{Mpc}}\right),
$$

where $n_{e}$ is the electron density. This means that effectively clusters are transparent for X-rays. As in the solar corona, this statement is not valid for the brightest X-ray resonant lines of the most abundant elements like iron. Gilfanov, Sunvaev \& Churazov (1987) have shown that the optical depth in the $6.7 \mathrm{keV}$ line of He-like iron in the brightest cluster A426 (Perseus) is larger than unity 11 and therefore effects of resonant scattering should be visible in the surface brightness distribution, element abundance variations or in the spectral shape of the line. It was immediately clear that all these effects are sensitive to the turbulent broadening of the line, making resonant scattering a unique tool to probe the characteristic amplitude of the gas velocities using telescopes with limited energy resolution. The impact of resonant scattering on surface brightness profiles has been studied via radiative transfer calculations (e.g. Gilfanov, Sunvaev \& Churazov 1987; Shigevama 1998; Churazov et al. 2004; Zhuravleva et al. 2010b) and searched for in observations of clusters, groups of galaxies and individual massive elliptical galaxies.

It has been realized that the scattering should also lead to a polarization of the same lines (Sazonov, Churazov \& Sunvaev 2002; Zhuravleva et al. 2010), with the polarization degree varying from $\sim 0$ at the center of a cluster to tens of per cent in the cluster outskirts (although the unpolarized continuum and nearby unpolarized lines will decrease this figure for an instrument with modest spectral resolution).

There have been numerous experimental studies aimed at detecting effects of resonant scattering in rich clusters and elliptical galaxies. For the Perseus cluster, an initial analysis suggested that resonant scattering effects were present (Molendi et al. 1998; Akimoto et al. 1999), although subsequent studies (Churazov et al. 2004; Gastaldello \& Molendi 2004) did not support these results (see also Ezawa et al. 2001; Dupke \& Arnaud 2001; Sanders et al. 2004). Several other clusters, including M87, Centaurus and A2199, were searched for resonant scattering effects (e.g Kaastra, Bleeker, \& Mewe 1999; Akimoto et al. 1999, 2000; Sanders \& Fabian 2006; Mathews, Buote, \& Brighenti 2001; Sakelliou et al.

\footnotetext{
1 For pure thermal broadening of the line.
} 
2002), with mixed results. For M87 and Perseus, resonant scattering was mentioned as one of the possible explanations of the central abundance dip Böhringer et al. 2001; Sanders et al. 2004).

In one object - the elliptical galaxy NGC4636 - most studies (Xu et al. 2002; Kahn et al. 2003; Werner et al. 2009; Havashi et al. 2009) agree that resonant scattering modifies the flux ratio of the Fe XVII lines at $15.01 \AA$ and $17.05 \AA$. The same study also found evidence, albeit at low statistical confidence, for resonant scattering in several other systems: NGC1404, NGC5813 and NGC4472.

In addition to the above mentioned studies several other ideas related to resonant scattering in hot cluster plasma have been proposed. Krolik \& Raymond (1988) (see also Sarazin 1989; Molnar, Birkinshaw, \& Mushotzky 2006) suggested to use measurements of the optical depth of the cluster plasma in lines together with the X-ray surface brightness to determine both the gas density and the linear size of the cluster. The known linear size can then be used for a cosmological distance-angular diameter test. Sazonov, Sunvaev, \& Cramphorn (2002) pointed out that scattering of continuum emission in a resonant line can be used to constrain the past X-ray luminosity of AGN in clusters of galaxies. Effects of resonant scattering of the cosmic X-ray background (CXB) were suggested to play a role in the line emissivity of the WHIM - warm-hot intergalactic gas (Churazov et al. 2001), provided that one can resolve a substantial fraction of point sources contributing to the CXB.

The structure of this paper is as follows: in section 2.1 we discuss the most promising resonant lines and calculate the optical depths for several objects; the impact of resonant scattering on line surface brightness profiles and on abundance measurements is discussed in section 3 in section 4 we analyze the sensitivity of the resonant scattering effects to the gas velocity field; in section 5 the polarization of X-ray lines is discussed; in section 6 we illustrate the effects of resonant scattering on the line shape; in section 7 we briefly discuss the possible uses of resonant scattering for cosmological tests (7.1), for searches of powerful AGN outbursts in the past (\$7.2) and for studying the WHIM (7.3).

\section{Basics of resonant scattering}

\subsection{Optical depth}

The cross section for scattering at the center of a resonant line can be written as

$$
\sigma_{0}=\frac{\sqrt{\pi} h r_{e} c f}{\Delta E_{D}}
$$

where $r_{e}$ is the classical electron radius and $f$ is the absorption oscillator strength of a given atomic transition and $\Delta E_{D}$ is the Doppler width of the line. In a plasma with a temperature typical of galaxy clusters, the line width is determined by the velocities of thermal and turbulent motions, rather than by the radiative width. For example, for the $6.7 \mathrm{keV}$ line of He-like iron the radiative width is $\sim 0.3 \mathrm{eV}$, while thermal broadening is $\sim 3 \mathrm{eV}$ for a $5 \mathrm{keV}$ gas. The Doppler width of the line is defined as

$$
\Delta E_{D}=E_{0}\left[\frac{2 k T_{e}}{A m_{p} c^{2}}+\frac{V_{\text {turb }}^{2}}{c^{2}}\right]^{1 / 2}
$$


where $A$ is the atomic mass of the corresponding element, $m_{p}$ is the proton mass and $V_{\text {turb }}$ is the characteristic turbulent velocity. $V_{\text {turb }}$ is often parametrized as $V_{\text {turb }}=$ $c_{s} M$, where $M$ is the Mach number 2 and the sound speed in the plasma is $c_{s}=$ $\sqrt{\gamma k T / \mu m_{p}}$, where $\gamma=5 / 3$ is the adiabatic index for an ideal mono-atomic gas and $\mu=0.61$ is the particle mean atomic weight. We can rewrite the previous expression as

$$
\Delta E_{D}=E_{0}\left[\frac{2 k T_{e}}{A m_{p} c^{2}}\left(1+1.4 A M^{2}\right)\right]^{1 / 2} .
$$

The optical depth of the cluster at the center of the line is then $\tau=\int \sigma_{0} n_{i} d l$, where $l$ is the distance along the photon propagation direction and $n_{i}$ is the number density of ions in the ground state of a given transition. For cluster conditions all ions are in the ground state (the frequency of collisions or any other excitation process are negligible compared to the radiative decay rate of the excited state) and $n_{i}=n_{p} Z \delta_{i}$, where $n_{p}$ is the density of protons, $Z$ the abundance of the element relative to hydrogen and $\delta_{i}$ is the fraction of the element in the appropriate ionization state. That is

$$
\tau=\int \frac{\sqrt{\pi} h r_{e} c f}{E_{0}\left[\frac{2 k T_{e}}{A m_{p} c^{2}}\left(1+1.4 A M^{2}\right)\right]^{1 / 2}} n_{p} Z \delta_{i} d l .
$$

Obviously, to have a large optical depth in the line one needs an astrophysically abundant element, a large oscillator strength of the transition, an appreciable ionization fraction of a given ion and a small line width. If the line width is dominated by thermal broadening then the lines of the heaviest elements have an advantage over lighter elements, since the thermal broadening scales as $1 / \sqrt{A}$.

Gilfanov, Sunvaev \& Churazov (1987) give a convenient expression for the optical depth (from the cluster center to the observer) at the midpoint of a Doppler-broadened resonance line when the isothermal gas density distribution can be approximated by a $\beta$-model $n=N_{0}\left[1+\left(\frac{r}{r_{c}}\right)^{2}\right]^{-3 / 2 \beta}$ :

$$
\begin{gathered}
\tau_{0}=\frac{\sqrt{\pi}}{2} \frac{\Gamma(3 \beta / 2-1 / 2)}{\Gamma(3 \beta / 2)} N_{z, 0} r_{c} \sigma_{0} \approx 2.7 \frac{\Gamma(3 \beta / 2-1 / 2)}{\Gamma(3 \beta / 2)} \frac{N_{0}}{10^{-3} \mathrm{~cm}^{-3}} \frac{Z}{Z_{\odot}} \delta_{i}(T) \\
\times \frac{r_{c}}{250 \mathrm{kpc}} \frac{\sigma_{0}\left(10^{7} \mathrm{~K}, \mathcal{M}=0\right)}{10^{-16} \mathrm{~cm}^{2}}\left[\frac{T}{10^{7} \mathrm{~K}}\left(1+1.4 A \mathcal{M}^{2}\right)\right]^{-1 / 2}
\end{gathered}
$$

where $\Gamma$ is the gamma-function; parameters of the $6.7 \mathrm{keV}$ line of FeXXV are used; and $Z / Z_{\odot}$ is the abundance of a given element relative to the solar abundance of iron. In Table1 1 we quote the optical depths of several promising lines for 3 objects, representing different mass limits - from an elliptical galaxy (NGC4636) to a rich cluster (Perseus). The optical depths were calculated using the observed temperature and density radial profiles. Pure thermal broadening of the lines was assumed.

From Table 1it is clear that different lines can be optically thick in objects having drastically different masses and temperatures. For objects with $T \geq 3 \mathrm{keV}$ (e.g. Perseus

\footnotetext{
2 This form of parametrization is rather arbitrary. For isotropic turbulence in a gas with $\gamma=5 / 3$, the value of $M=1$ corresponds to the energy in turbulent motions being equal to 0.83 times the thermal energy density $\frac{1}{\gamma-1} \frac{\rho}{\mu m_{p}} k T$.
} 
Table 1 Oscillator strength, Rayleigh scattering weights and optical depth of the most promising X-ray lines for the elliptical galaxy NGC 4636 and the M87/Virgo and Perseus (A426) clusters. The optical depths were calculated using the observed temperature and density profiles and assuming the flat abundance profile $Z=0.5 Z_{\odot}$ (relative to the Solar abundances of Anders \& Grevesse (1989)), collisional ionization equilibrium and pure thermal broadening of the lines.

\begin{tabular}{rcccccc}
\hline Ion & $E, \mathrm{keV}$ & $f$ & $w_{2}$ & $\tau$, NGC 4636 & $\tau$, Virgo/M87 & $\tau$, Perseus \\
\hline O VIII & 0.65 & 0.28 & 0.5 & 1.2 & 0.34 & 0.19 \\
Fe XVIII & 0.87 & 0.57 & 0.32 & 1.3 & 0.0007 & $1.5 \cdot 10^{-7}$ \\
Fe XVII & 0.83 & 2.73 & 1 & 8.8 & 0.0005 & $2.8 \cdot 10^{-8}$ \\
Fe XXIII & 1.129 & 0.43 & 1 & 0.016 & 1.03 & 0.16 \\
Fe XXIV & 1.168 & 0.245 & 0.5 & 0.002 & 1.12 & 0.73 \\
Fe XXV & 6.7 & 0.78 & 1 & 0.0002 & 1.44 & 2.77 \\
\hline
\end{tabular}

cluster) the He-like iron line at $6.7 \mathrm{keV}$ is a likely winner; while for cool systems (e.g. NGC4636 with $T \sim 0.6 \mathrm{keV}$ ) the Ne-like iron line at $0.83 \mathrm{keV}$ has the largest optical depth.

\subsection{Phase function}

For any transition, the resonant scattering can be represented as a combination of two processes: isotropic scattering with a weight $w_{1}$ and Rayleigh scattering with a weight $w_{2}=1-w_{1}$ (Hamilton 1947; Chandrasekhar 1950). The weights $w_{1}$ and $w_{2}$ depend on the total angular momentum $j$ of the ground level and on the difference between the total angular momenta of the excited and ground levels $\Delta j(= \pm 1$ or 0$)$. The expressions for the weights are given by Hamilton (1947). For a subset of the most promising lines the $w_{2}$ values are given in Table 1

If the radiation is initially unpolarized, then the probability for a photon to scatter into a unit sold angle at an angle $\theta$ with respect to the incident direction is

$$
P(\mu)=\frac{1}{4 \pi}\left[w_{1}+\frac{3}{4}\left(1+\mu^{2}\right) w_{2}\right],
$$

where $\mu=\cos \theta$.

If polarization has to be accounted for (see $\$ 5$ ), then the scattering matrix of resonant scattering can be written as

$$
\left(\begin{array}{l}
I_{l}^{\prime} \\
I_{r}^{\prime} \\
U^{\prime}
\end{array}\right)=\frac{1}{4 \pi}\left[\frac{1}{2} w_{1}\left(\begin{array}{ccc}
1 & 1 & 0 \\
1 & 1 & 0 \\
0 & 0 & 0
\end{array}\right)+\frac{3}{2} w_{2}\left(\begin{array}{ccc}
\mu^{2} & 0 & 0 \\
0 & 1 & 0 \\
0 & 0 & \mu
\end{array}\right)\right]\left(\begin{array}{c}
I_{l} \\
I_{r} \\
U
\end{array}\right),
$$

where $I_{l}, I_{r}, U$ are the Stokes parameters as defined by Hamilton (1947); Chandrasekhar (1950).

For the changes in the surface brightness (see $\$ 3$ ), the difference between the isotropic and Rayleigh phase functions is not very important, while it is crucial if one needs to evaluate the degree of polarization (see \$5). Isotropic scattering does not produce polarization. Instead it "erases" information on the initial direction and orientation of the electric vector in the "memory" of the scattered photon. On the contrary, Rayleigh scattering changes the polarization state of the radiation field. 

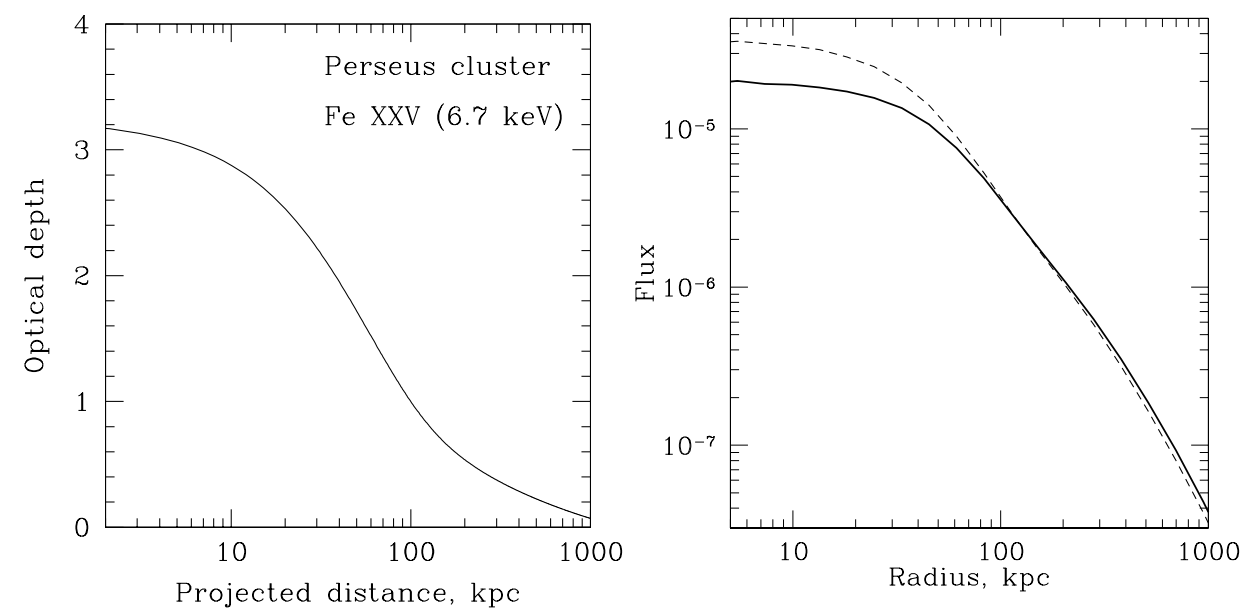

Fig. 1 Left: Optical depth of the Perseus cluster in the $6.7 \mathrm{keV}$ FeXXV line over the range of radii from $r$ to $\infty$. For $r_{1} \approx 70 \mathrm{kpc}$ the optical depth is of order 1 . To a first approximation the surface brightness is expected to be only weakly disturbed by resonant scattering for projected radii $R \gg r_{1}$. For smaller projected radii the surface brightness profile is expected to be flat. Right: Radial profiles of the He-like iron $K_{\alpha}$ line with (thick solid line) and without (dashed line) resonant scattering in the Perseus cluster. Resonant scattering suppresses the line intensity in the core and redistributes line photons to larger radii. Pure thermal broadening and a flat radial abundance profile were assumed in this calculation. Adapted from Churazov et al. (2004).

\section{Impact on surface brightness profile and abundance}

Since the density of the gas declines faster than $\sim r^{-1}$ in the outer regions of all clusters, the optical depth of the outer regions in any line is small (see Fig. 1 left). We therefore do not expect strong modifications of the line surface brightness profile at large projected distances from the cluster center (see Fig. 1 right). The central part on the contrary is dense and in the region where the optical depth is of order unity one can expect that resonant scattering will erase all features in the surface brightness, leading to a flattened (see Fig. 1 right) surface brightness distribution (Gilfanov, Sunyaev \& Churazov 1987). Due to the conservative nature of resonant scattering, the photons removed from the line of sight going through the cluster center are re-distributed to larger projected distances. This effect causes a slight increase of the surface brightness at $R>r_{1}$ (see Fig. 1 right). Since the surface brightness in the continuum is not modified, the resonant scattering causes a decrease of the equivalent width of the line in the cluster center and a slight increase of the equivalent width in the outer regions. This could cause an apparent decrease in the abundance of heavy elements in the central part of the cluster if resonant lines are used to measure it (Gilfanov, Sunvaev \& Churazov 1987).

This effect was suggested to play a role in the spurious "abundance holes "in cluster centers (e.g. Böhringer et al. 2001; Sanders et al. 2004), although other effects such as the presence of multi-temperature plasma may be more important in these environments (e.g. Buote 2000). 
Note that optically thin lines are not modified by resonant scattering and therefore the observed spectrum might have line ratios different from the expected values for an optically thin thermal plasma. This implies that one can use the line ratios (the flux in an optically thick line divided by the flux in an optically thin line) rather than the line equivalent width (i.e. the ratio of the flux in the optically thick line to the spectral intensity of the continuum). The ideal situation is when two lines from the same ion are used, since in this case many issues related to the modeling of the ionization state can be avoided.

A set of simple expressions was derived by Gilfanov. Sunvaev \& Churazov (1987); Sazonov, Churazov \& Sunyaev (2002) for model gas density distributions and in the limit of $\tau \ll 1$ for the isotropic and Rayleigh phase functions. These expressions can be used for making order of magnitude estimates, while for real systems it is necessary to solve a radiative transfer problem to predict the shape of distortions, especially when substantial temperature gradients are present, which affect the ionization state and therefore the radial distribution of ions.

Overall the resonant scattering has been searched for in a number of objects, although clear evidence for the effect was found only in few systems - for the Ne-like iron (Fe XVII) line at $15.01 \AA$ in NGC4636 (Xu et al. 2002; Kahn et al. 2003; Werner et al. 2009; Hayashi et al. 2009) and for NGC1404, NGC5813 and NGC4472 (Werner et al. 2009). Nevertheless, the magnitude of the effect (modification of the surface brightness distribution in the line) is well within the capabilities of modern X-ray telescopes. In fact, one can use the absence of resonant scattering effects to infer the properties of the gas velocity field, as discussed in the next section.

\section{Sensitivity to the velocity field}

If one accurately measures the temperature of the intracluster medium (ICM), then the line ratios of (optically thick) resonant and (optically thin) non-resonant lines can be used as a powerful tool to measure the line widths and therefore to get information on the velocity field, which is difficult to obtain by other means. Given that the optical depth sensitively depends on the turbulent broadening of resonant lines, especially for heavy elements like iron, the comparison of the fluxes of e.g. iron and nickel lines could be used to place constraints on the level of turbulence in clusters (Churazov et al. 2004). For the Perseus cluster, the XMM-Newton spectra of the central region do not show a suppression of the resonant $6.7 \mathrm{keV}$ line of iron relative to a much more optically thin nickel line when the APEC (Smith et al. 2001) plasma emission model is used, although one should expect the $6.7 \mathrm{keV}$ line of He-like iron to be suppressed in the core if the iron line were only thermally broadened. Indeed, without additional line broadening the optical depth of the $6.7 \mathrm{keV}$ line is $\sim 3$ (see Table 1). Therefore, the line in the central $\sim 1^{\prime}$ region must be suppressed by a factor of about 1.7 (see Fig. 2), in contrast with observations.

Even more robust constraints are expected if optically thick and optically thin lines of the same element or even better of the same ion are used. Werner et al. (2009) obtained high resolution spectra of the giant elliptical galaxy NGC 4636 using the grating spectrometers on the XMM-Newton satellite. A detailed study of the spectra proved that the Fe XVII line at $15.01 \AA$ is suppressed (Fig. 3) only in the dense core and not in the surrounding regions, while the line of Fe XVII at $17.05 \AA$ is optically thin and is not suppressed. Werner et al. (2009) modeled the radial intensity profiles 

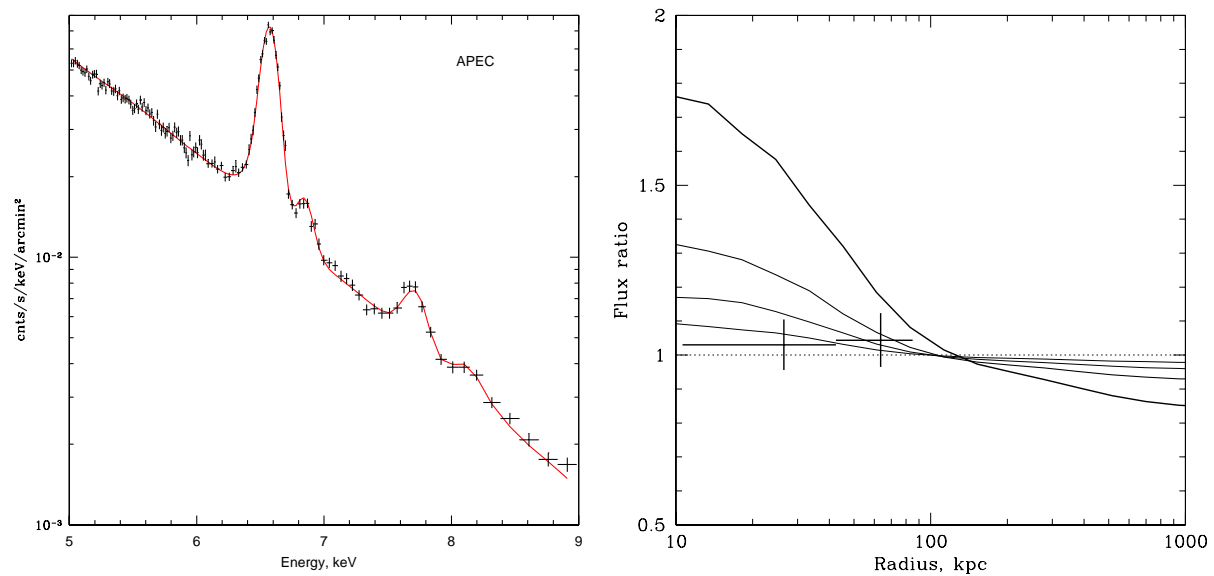

Fig. 2 Left: 5-9 keV spectrum of the $30^{\prime \prime}$ to $2^{\prime}$ annulus centered on NGC 1275 and fitted with APEC (Smith et al. 2001) models. Right: Influence of turbulence on the strength of the resonant scattering effect. The curves show the expected ratio of the optically thick and optically thin lines calculated for effective Mach numbers of $0,0.25,0.5$ and 1 (from top to bottom). For comparison, crosses show the observed ratio of heavy element abundances obtained ignoring parts of the spectrum containing the $6.7 \mathrm{keV}$ and $7.9 \mathrm{keV}$ complexes, respectively. Given that the resonant $K_{\alpha}$ line of He-like iron contributes about $50 \%$ to the $6.6-6.8 \mathrm{keV}$ complex of lines, the measured abundance ratio is consistent with the curves for Mach number $\geq 0.5$. Adapted from Churazov et al. (2004).

of the optically thick line, accounting for the effect of resonant scattering for different values of the characteristic turbulent velocity. Comparing the model to the data, it was found that the isotropic turbulent velocities on spatial scales smaller than $\approx 1 \mathrm{kpc}$ are less than $100 \mathrm{~km} / \mathrm{s}$ and the turbulent pressure support in the galaxy core is smaller than 5 per cent of the thermal pressure at the 90 per cent confidence level.

While there is still a bit of a controversy in the results, which definitely suffer from the limited energy resolution of the present-day CCDs or from the finite size of the emitting region for RGS data, it is clear that the magnitude of the effect is within the reach of the current generation of telescopes and the situation will improve in future.

The relation between the resonant scattering effects and the velocity field can be used to test even more subtle effects, such as e.g. anisotropy of gas motions. Zhuravleva et al. (2010b) showed that for pure radial motions versus pure tangential motions the amplitude of the effect can change by a factor of 1.5 for realistic values of the velocity amplitude (see the left panel in Fig. 4). Clearly, tangential gas motions do not change the optical depth in the line (the right panel, Fig. 4) calculated along the radial direction. Photons coming from the cluster central region are expected to make the largest contribution to the scattered flux. Since they move essentially along the radius, tangential gas motions do not affect the probablity of these photons to be scattered. Therefore, to a first approximation the presence of pure tangential motions does not affect much the magnitude of the resonant scattering effect. Radial motions, on the contrary, strongly affect the optical depth for the photons coming from the cluster center (the right panel, Fig. 4) and decrease the efficiency of the resonant scattering. The net result of the simulations by Zhuravleva et al. (2010b) is that the conversion 

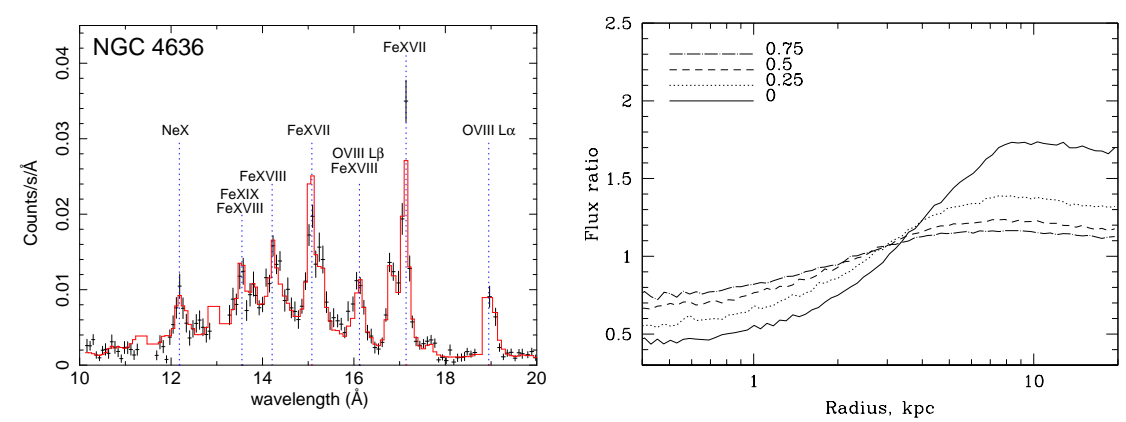

Fig. 3 Left: $X M M$-Newton RGS spectra extracted from a $0.5^{\prime}$ wide region centered on the core of NGC 4636. The full line indicates the best optically thin single-temperature plasma model fit. The 13.8-15.5 $\AA$ part of the spectrum, where the strongest Fe XVII and Fe XVIII resonance lines are present, was excluded from these spectral fits. Right: Simulated radial profiles of the ratio of the $15.01 \AA$ line intensities calculated with and without the effects of resonant scattering, for isotropic turbulent velocities corresponding to Mach numbers 0.0, 0.25, 0.5 , and 0.75 and a flat abundance profile. Adapted from Werner et al. (2009).

of the observable magnitude of the flux suppression to the characteristic amplitude of gas motions depends on the anisotropy of the velocity field. In many situations the assumption of isotropic stochastic motions is reasonable. But there are cases, e.g. spherical shocks/sound waves propagating through the ICM (e.g. Forman et al. 2007), when strong anisotropy of the gas motions is expected.

Zhuravleva et al. (2010b) also tested the impact of the spatial scales of motions on scattering, showing that suppression of the line flux towards the center of the cluster is insensitive to the presence of gas motions on large scales. This is easy to understand since small scale motions directly affect the line width and the optical depth of the line. If gas motions are present on scales larger than the size of the central region where $\tau$ is of order unity, then these motions can be interpreted as a motion of the whole central region. The optical depth and the magnitude of the flux suppression remain unaffected.

\section{Polarization due to resonant scattering}

Scattering in strong resonant lines that have a non-zero weight of the Rayleigh phase function can lead to linear polarization of scattered radiation (see section 2.2). To produce net polarization during the Rayleigh scattering of initially unpolarized light a quadrupole component has to be present in the radiation field. It appears quite naturally in galaxy clusters due to (i) the centrally peaked gas density distribution and (ii) differential gas motions (see Fig. 5).

In the cluster center, the radiation field is isotropic (for a spherical cluster). Therefore, due to the symmetry radiation will be unpolarized. However, at larger distances from the center a quadrupole component appears in the radiation field due to the strong dominance of the flux coming from the cluster center compared to all other directions. 

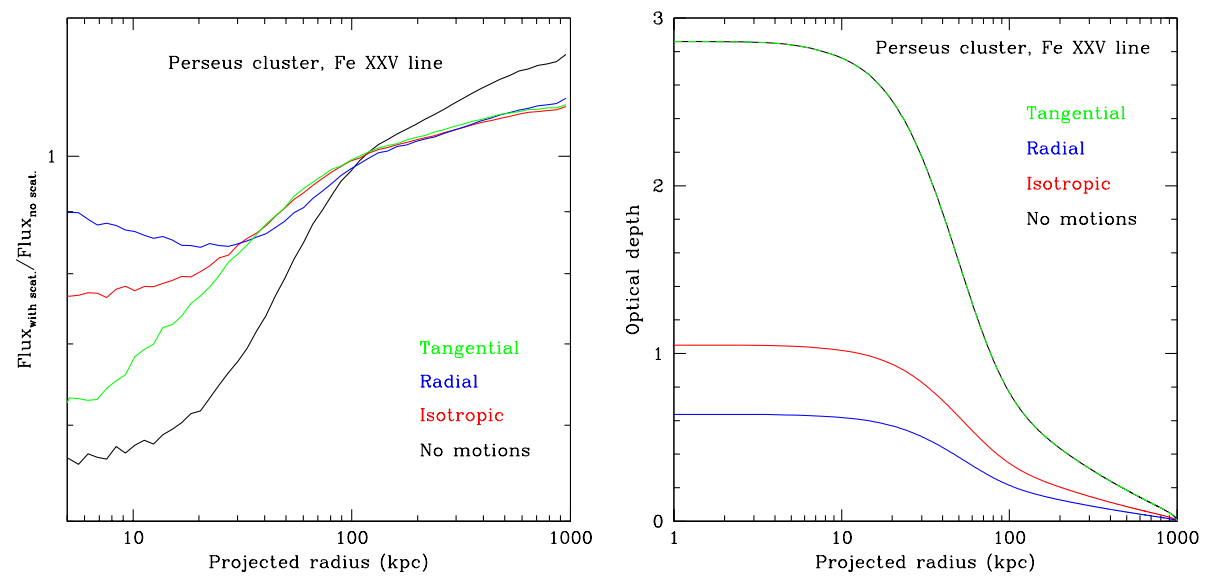

Fig. 4 Left: Impact of the anisotropy of stochastic gas motions on the ratio of the fluxes in the Fe XXV line at $6.7 \mathrm{keV}$ in the Perseus cluster calculated with and without allowance for scattering. The RMS of the 3D velocity field is $500 \mathrm{~km} / \mathrm{s}$ in all cases (except for the case with no gas motions). Adapted from Zhuravleva et al. (2010b). Right: Optical depth of the Perseus cluster in the $6.7 \mathrm{keV} \mathrm{FeXXV}$ line over the range of radii from $\mathrm{r}$ to $\infty$, calculated assuming different directions of stochastic gas motions.

These photons coming from the center give rise to the polarization of the scattered radiation (see Fig. 5] the left panel). Obviously, the polarization plane is perpendicular to the radius.

The polarization degree was first calculated by Sazonov, Churazov \& Sunyaev (2002) (see also Zhuravleva et al. 2010) for the Perseus and Virgo clusters (see Fig. 6). For the Perseus cluster, the polarization degree was calculated in the He-like iron line at $6.7 \mathrm{keV}$, which has an optical depth $\sim 3$. The polarization is zero at the center of the cluster (as expected from the symmetry of the problem) and increases rapidly with distance from the center, reaching $\sim 10$ per cent at $\sim 1 \mathrm{Mpc}$ (Fig. 6] the left panel). The polarization degree in the Virgo cluster in the most promising lines is a few per cent (Fig. 6, the right panel).

The assumption of spherical symmetry is a strong one, since clusters are rarely spherical. Depending on the characteristics of the substructure in the azimuthal density and temperature distributions the polarization degree can change either way: increase or decrease even if one neglects non-zero gas velocities. When working with simulated clusters we see variations of the polarization degree by as much as factor of two when 3-dimensional density and temperature distributions are replaced with the azimuthly averaged values.

Gas motions affect both the amplitude and the direction of polarization. If a gas lump is moving relative to the whole cluster with high speed, such that the Doppler shift of the line exceeds the line width (i.e. the line leaves the resonance), then scattering will not occur along the direction of motion, while photons coming from the perpendicular direction will be scattered, producing net polarization. The sketch of one of the possibilities is shown of Fig. 5 (the right panel).

Zhuravleva et al. (2010), used full 3D models of galaxy clusters taken from cosmological simulations (Dolag et al. 2008; Springel, Yoshida, \& White 2001) to calculate 

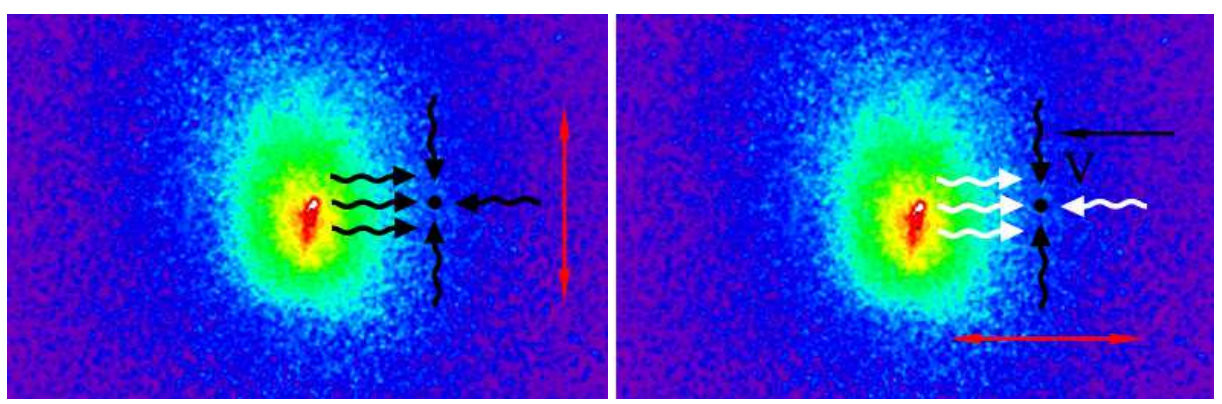

Fig. 5 Sketch of the problem. Polarization arises due to the quadrupole component in the unpolarized radiation field produced by a centrally peaked gas density distribution (the left panel) and/or differential gas motions (the right panel). The line of sight is perpendicular to the picture plane. Left panel: scattering occurs at the edge of the cluster (the black dot), the black wavy arrows show photons coming from different directions and participating in scattering; the red arrow shows the direction of polarization of the scattered radiation (the orientation of the electric vector). Right panel: the black dot shows the place where scattering occurs, the black straight arrow shows the direction of motion of the gas lump with velocity $\mathbf{V}$ relative to the whole cluster, the black wavy arrows show photons participating in the scattering while the white wavy arrows show photons that will not be scattered due to gas motions (the line leaves the resonance in the direction of motion), the red arrow shows the direction of polarization of the scattered radiation.

the polarization degree with an account for gas motions. For one of the most massive simulated clusters ( $g 8$ in the sample of Dolag et al. (2008)) the polarization degree in the He-like iron line reaches $\sim 25$ per cent at a distance of $\sim 500 \mathrm{kpc}$ from the center if no turbulent motions are present (Fig. 7 the left panel). Inclusion of gas motions substantially decreases the polarization down to $\sim 10$ per cent (Fig. 7 the right panel) within the same region. They also made calculations for a bullet-like cluster ("g72" cluster in the simulated sample of Dolag et al. (2008)), showing that the polarization degree is less that 7 per cent within the region where interaction of two sub-clusters occurs.

When polarization in lines is considered, one should take into account the contamination of the polarized flux in the resonant line by the unpolarized emission in the neighboring spectral continuum and nearby lines. The effect is severe. For example, for the $6.7 \mathrm{keV}$ line an energy resolution of $\sim 50 \mathrm{eV}$ leads to a factor of 2 drop in the degree of polarization. Also, at large distances from the cluster center unpolarized CXB emission starts to dominate.

Currently new concepts of X-ray polarimeters are under discussion (see Muleri et al. 2009; Soffitta et al. 2008; Costa et al. 2008, e.g.). Galaxy clusters are promising but challenging targets for future polarimetric observations. Polarimeters should have good energy resolution (better than $100 \mathrm{eV}$ ), which is hardly reachable with the nearest future X-ray polarimeters. In addition, arcminute angular resolution is needed to resolve nearby clusters. Naturally, a large field of view and effective area are required to collect large numbers of photons (see Zhuravleva et al. 2010).

\section{Impact on the line shape}

The optical depth given by eq. 7 corresponds to the center of the line, i.e. to a photon energy equal to the line energy $E_{0}$. If a photon is born in the wing of the line, then 

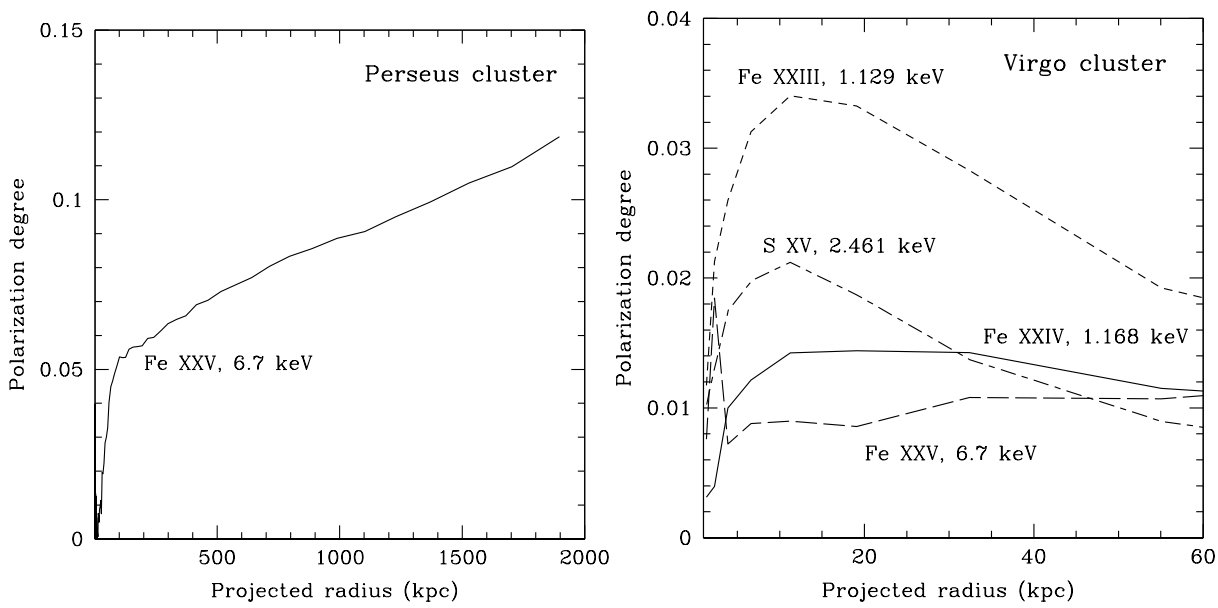

Fig. 6 Left panel: Expected polarization degree as a function of projected distance from the center of the Perseus cluster in the most prominent resonant line of Fe XXV at $6.7 \mathrm{keV}$ (calculated assuming constant iron abundance). Right panel: Expected polarization degree as a function of projected distance from the center of the Virgo/M87 cluster in the most prominent resonant lines.
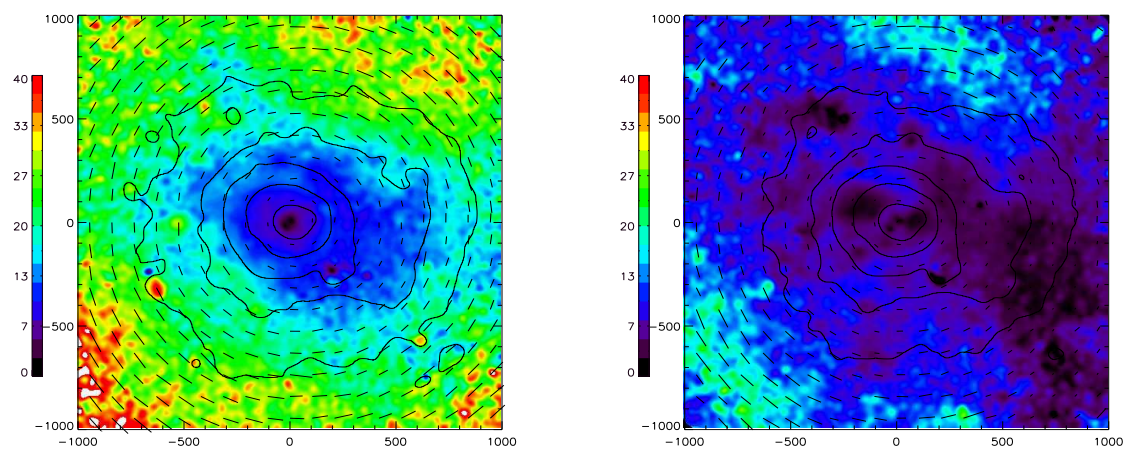

Fig. 7 Polarization degree of the simulated massive cluster g8 (Dolag et al. 2008) in the $\mathrm{K}_{\alpha}$ line of Fe XXV at $6.7 \mathrm{keV}$. The polarization degree was evaluated as $P=\sqrt{Q^{2}+U^{2}} / I$. I is the total intensity, including scattered and direct emission. The colors in the images denote the polarization degree in per cent. The short dashed lines show the orientation of the electric vector. Contours (factor of 4 steps in intensity) of the X-ray surface brightness in the chosen line are superposed. The size of each picture is $2 \times 2 \mathrm{Mpc}$. The left panel shows the case of gas being at rest. The right panel shows the case of the gas velocities obtained in the simulations. 

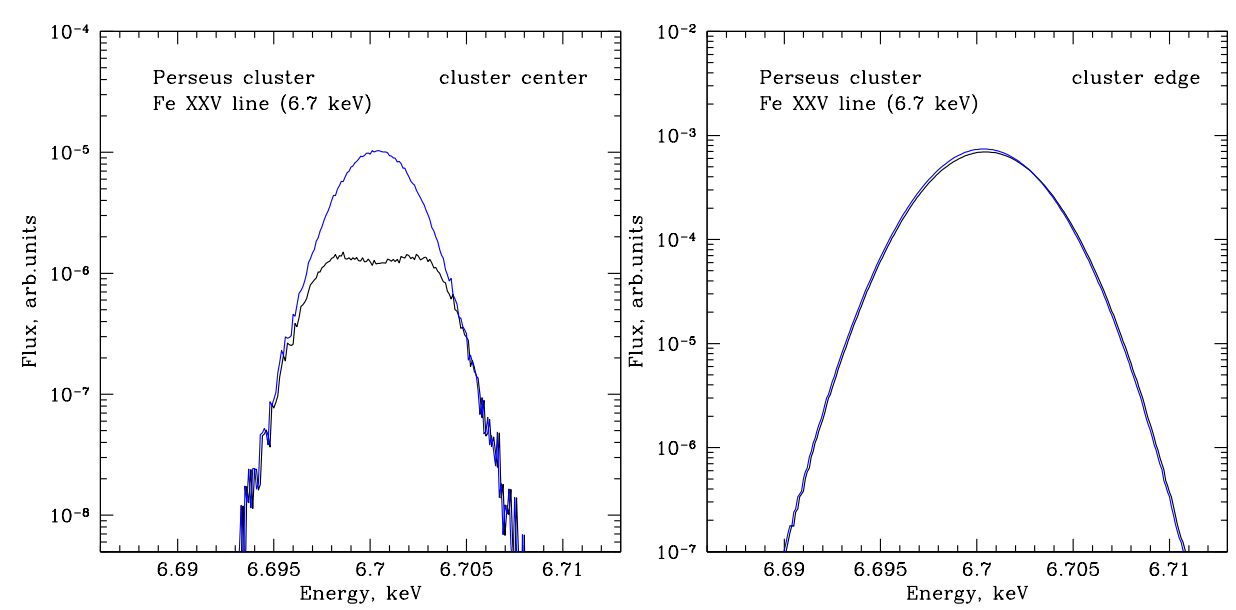

Fig. 8 Simulated spectra of the $6.7 \mathrm{keV}$ line emerging from the core of the Perseus cluster (left) and at a projected distance of $800 \mathrm{kpc}$ (right). Lines are broadened only due to thermal motions of ions. The calculations were done with (black curves) and without (blue curves) accounting for resonant scattering. The line in the cluster outskirts (right panel) is broader due to the higher temperature in the outer regions of the Perseus cluster.

the optical depth will be lower,

$$
\tau(E)=\tau_{0} e^{-\left(\frac{E-E_{0}}{\Delta E_{D}}\right)^{2}} .
$$

If $\tau(E) \ll 1$, then such photons will leave the cluster without scattering, while photons with $E \sim E_{0}$ will be scattered from the line of sight going through the cluster center. It is therefore clear that resonant scattering should affect the shape of optically thick lines. The spectral profiles of the He-like iron line at $6.7 \mathrm{keV}$ calculated in the core of the Perseus cluster and at a projected distance of $\sim 800 \mathrm{kpc}$ are shown in Fig. 8 The calculations were done with (black curves) and without (blue curves) allowance for scattering. The lines are broadened only due to thermal motions of ions. One can notice the suppression of the emission in the line center (left panel) caused by resonant scattering: photons with the energy close to the line central energy are scattered from the line of sight because of the large optical depth, while photons born in the wings escape freely. At the edge of the cluster the optical depth is small and the line profiles almost match each other.

\section{Various extensions}

\subsection{Cosmological tests}

The effects of resonant scattering are proportional to the optical depth of the cluster (see eq. 6), which scales linearly with the size of the cluster $l$ and the gas density $n_{e}: \tau \propto n_{e} l \times F(T, Z)$, where the factor $F(T, Z)$ accounts for the element abundance, ionization equilibrium and the line broadening. If another characteristic of the cluster (with a different dependence on $l$ and $n_{e}$ ) is known, then both $l$ and $n_{e}$ can be determined simultaneously. For instance, one can use the X-ray surface brightness, which is 
proportional to $n_{e}^{2} l \times W(T, Z)$, where the $W(T, Z)$ factor accounts for the temperature dependent emissivity and the gas metallicity. Once $l$ of a spherically symmetric cluster is known, one can immediately perform an angular diameter-redshift test, analogously as is done using a combination of the cosmic microwave background distortions (the Sunyaev-Zel'dovich effect) and the X-ray surface brightness. Thus a simple cosmological test based solely on X-ray data is possible. This is an attractive possibility since such a test can be performed by a single X-ray telescope in a single observation.

Several flavors of the same technique have been proposed. Krolik \& Ravmond (1988) and Sarazin (1989) suggested to look for absorption lines in the spectrum of an $\mathrm{X}$-ray emitting background quasar. The equivalent width of the strongest absorption lines can be of order several eV. Another possibility is to use the distortions of the cluster emission itself, using the polarization (Sazonov, Churazov \& Sunvaev 2002) or shape of emission lines (Molnar. Birkinshaw, \& Mushotzkv 2006), or directly the radial variations of the equivalent width or the flux ratios of optically thick and thin lines (as discussed by Gilfanov, Sunyaev \& Churazov 1987). Some variants of these methods are within the reach of currently operating X-ray telescopes, but the uncertainties in line widths and the complex spectra of the central regions of cool core clusters complicate the analysis. Significant progress is expected with the launch of X-ray micro-calorimeters with energy resolution of a few eV (e.g. Mitsuda et al. 2008). They will simultaneously constrain the presence of stochastic gas motions and allow for accurate measurements of line shapes (see e.g. Fig [8).

\subsection{AGN X-ray echo in lines}

To better understand how massive black holes grow in galactic nuclei, one would like to have information on AGN variability on time scales much longer than the several tens of years probed by historic light curves. While the behavior on the longest time scales (hundreds of millions of years) is determined by the supply of gas (e.g. triggered by a merger) from the outer regions of a galaxy to the central kiloparsec, AGN can switch on and off on intermediate time scales for a number of reasons, e.g. due to instabilities in the accretion disk or due to feedback of the growing massive black hole on the surrounding medium. Clearly, such long-term phenomena can only be studied by means of indirect observations. An attractive idea is to observe X-ray radiation which was emitted in the past by a galactic nucleus and later scattered in our direction by ambient gas (Sazonov, Sunyaev, \& Cramphorn 2002). Cluster dominant elliptical galaxies are the most promising targets for such observations, since their extended gas haloes make it possible to probe time scales up to a few million years. Since AGN radiation is much harder than the thermal emission of intracluster gas, one possibility is to search for AGN "echoes" at energies $E \gg k T /(1+z)$ ( $T$ being the gas temperature and $z$ the redshift of the object). An additional significant advantage is provided by resonant X-ray lines, since the scattered/intrinsic flux ratio is expected to be larger by a factor of 3-10 in a resonance line than in the neighboring continuum.

Sazonov, Sunvaev, \& Cramphorn (2002) assessed the level of constraints that could be derived from future observations on the past X-ray luminosity of M87 and Cyg A (see Fig. 9), giant elliptical galaxies residing in the central regions of two nearby clusters of galaxies. For instance, scattered line radiation should be detectable from the Virgo cluster if the X-ray luminosity of M87 was a few $10^{44} \mathrm{erg} \mathrm{s}^{-1}$ (i.e. three orders of magnitude brighter than presently) until a few $10^{5}$ years ago. The same method can 

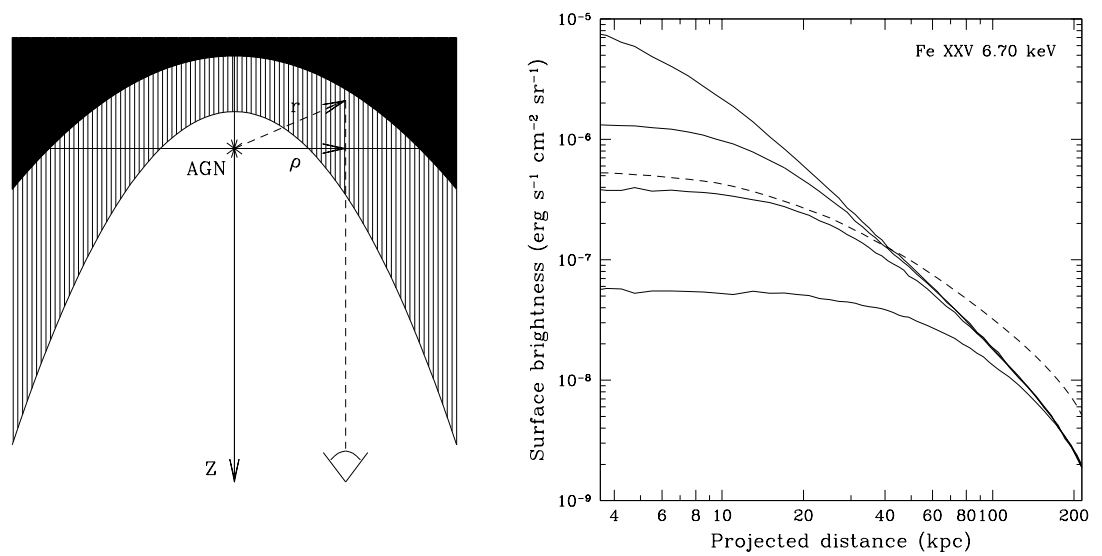

Fig. 9 Left: Illustration of the delayed X-ray line echo of an AGN outburst in a galaxy cluster. Locations with equal travel time from the AGN to the observer lie on a paraboloid. In the switch-off case (the AGN was bright for a long time until some instant in the recent past), the cluster plasma located in the grey and black regions is scattering the X-ray radiation emitted by the AGN. In the case of a short AGN flare, only the grey area is filled by photons and contributing to the scattered radiation. A possible path for a photon scattered into the observer's line of sight is marked by a dashed line. Right: M87/Virgo surface brightness of scattered AGN radiation (solid lines) in the $6.70 \mathrm{keV}$ line of He-like iron at times (from top to bottom) of 5, 50, 100 and 250 thousand years after an AGN switch-off. The dashed line shows the intrinsic surface brightness profile of the cluster gas in the $6.70 \mathrm{keV}$ line. The AGN luminosity was $L_{X}=0.01 L_{E d d}$ before switch-off. Adapted from Sazonov, Sunyaev, \& Cramphorn (2002).

also be applied to groups of galaxies and isolated giant elliptical galaxies, which too are large reservoirs of hot gas capable of scattering AGN radiation. Of course, the testable time scales are shorter (up to a few times $10^{5}$ years) than for clusters of galaxies.

\subsection{Warm-Hot Intergalactic Medium (WHIM) and blazars}

Resonant scattering may be important not only in the dense gas inside a cluster, but also in a much more tenuous warm intercluster medium in filaments. In the local Universe such structures have linear sizes of $5-10 \mathrm{Mpc}$, temperatures of $\sim 10^{5}$ to $\sim 10^{7}$ $\mathrm{K}$ and density $\delta=10-100$ times larger than the mean baryonic density of the Universe $\left(\rho_{\text {bar }}=3.610^{-31} \mathrm{~g} \mathrm{~cm}^{-3}\right)$. Simulations suggest that these structures may account for a significant or even dominant fraction of the baryons at $z=0$ (e.g. Cen \& Ostriker 1999).

The low density of filaments makes direct detection of their thermal emission extremely difficult. If there is a bright QSO then one can try to search for absorption lines using high resolution spectroscopy (e.g Nicastro et al. 2005), although it is not clear if existing data have already provided a robust detection of truly diffuse gas (e.g Rasmussen et al. 2007). If one is looking for WHIM in the directions where no bright QSO is present, then one can still use a combined signal of numerous background sources (and search for an absorption feature) or instead look "between" the 

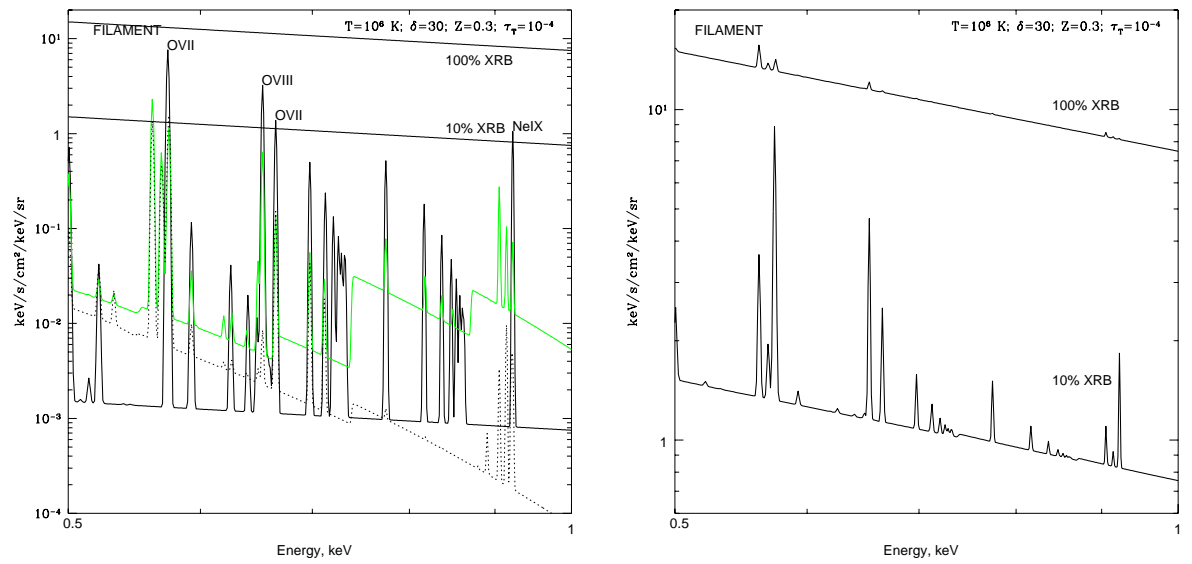

Fig. 10 Left: The X-ray spectrum of warm gas with an overdensity of $\delta=30$, a temperature of $10^{6} \mathrm{~K}$, a Thomson optical depth of $10^{-4}$ and a metallicity of $30 \%$ solar. These conditions should be typical for a filamentary structure in the warm IGM. The dotted curve shows the emission spectrum due to collisional excitation and ionization. For the green curve photoionization by the CXB was taken into account. The black solid curve shows Thomson plus resonantly scattered radiation. The upper solid line is the intensity of the CXB from the same region. The lower solid line shows $10 \%$ of the CXB intensity (the level to which discrete sources may be removed). The spectra were convolved with a Gaussian with a FWHM of $2 \mathrm{eV}$. Right: The upper curve shows the total spectrum of a region of the sky containing a filament with Thomson optical depth of $10^{-4}$. This spectrum is a sum of the thermal emission of the filament, scattered CXB and emission of the background sources, part of which is either absorbed or scattered by the filament. Note that resonance scattering does not change the CXB flux and only emission lines due to the thermal emission of the filament are visible in this spectrum. The lower curve shows the spectrum of the same region if $90 \%$ percent of the background is resolved into compact sources and removed. Note that the most prominent OVII line at 0.57 $\mathrm{keV}$ becomes visible only in this spectrum. In the hypothetical case that all CXB is resolved and removed the residual spectrum should be equal to the sum of the thermal emission of the filament and scattered CXB emission. These two components are shown in the left panel. Adapted from Churazov et al. (2001).

background sources. As pointed out by Churazov et al. (2001) the scattering of X-ray background photons in He and H-like ions of heavy elements can exceed the "local" thermal emission of a filament by a factor of a few or more. Due to the conservative nature of resonant scattering, this resonantly scattered radiation can only be identified if a significant fraction of the CXB is resolved and removed. While the combined spectrum of the resolved sources will contain X-ray absorption features, the residual background will contain corresponding emission features with the (on average) same intensity. At the relevant densities and temperatures, the lines of $\mathrm{He}$ and $\mathrm{H}$-like oxygen at 0.57 and $0.65 \mathrm{keV}$ are most promising. These lines (which have a typical width of $\sim 1-2 \mathrm{eV}$ ) may contain up to $50 \%$ of the total $0.5-1 \mathrm{keV}$ emission of the filament (Fig. 10). Note that the detection of WHIM in absorption using a combined signal of background objects and the detection of a line in emission using a part of the image without bright sources impose different requirements on the telescope characteristics. In the former case, the energy resolution is crucial, since the goal is to detect a weak absorption line in the combined spectrum of all resolved sources - in practice the best results will be achieved if the energy resolution is better than the equivalent width of 
the absorption line. In the latter case, the same total flux is compared with the unresolved background - the low internal background is crucial in this case, while superb energy resolution is less of an issue. In both cases the angular resolution has to be good enough to resolve $\geq 50 \%$ of the CXB.

On average up to a few percent of the soft CXB could be resonantly scattered by this phase of the IGM and resonantly scattered photons should account for a significant fraction of the truly diffuse background at low energies. Close to bright X-ray sources such as galaxy clusters or AGN the flux of scattered radiation will be further enhanced. From this point of view, off-line blazars are the most promising illuminating sources. The scattered emission from AGN may also constrain the duration of the active phase of these objects analogously to what is discussed in $\$ 7.2$.

\section{Conclusions}

Resonant scattering in the brightest X-ray emisson lines leads to a number of characteristic observational features, inluding distortions in the surface brightness, modifications of the line spectral shape and polarization. The sensitivity of the resonant scattering effects to the gas velocity field makes them a promising tool for IGM studies. Resonant scattering can also be used for the basic cosmological angular diameter/redshift test, as a tracer of a very rarefied IGM and as a diagnostic of past outbursts from AGN. While the distortions in line surface brightness profiles are within the reach of modern $\mathrm{X}$-ray telescopes, future high energy resolution and polarimetric missions will be able to fully exploit resonant scattering as a diagnostic tool.

Acknowledgements We are grateful to Klaus Dolag, William Forman, Nail Inogamov, Dmitry Nagirner, Leonid Vainshtein and Norbert Werner for useful discussions.

\section{References}

Acton L. W., 1978, ApJ, 225, 1069

Akimoto F., Furuzawa A., Tawara Y., Yamashita K., 1999, AN, 320, 283

Akimoto F., Furuzawa A., Tawara Y., Yamashita K., 2000, AdSpR, 25, 603

Anders E., Grevesse N., 1989, GeCoA, 53, 197

Böhringer H., et al., 2001, A\&A, 365, L181

Buote D.A. 2000, ApJ, 539, 172

Cen R., Ostriker J. P., 1999, ApJ, 514, 1

Chandrasekhar S., 1950, Radiative Transfer, Oxford, Clarendon Press, 1950

Churazov E., Haehnelt M., Kotov O., Sunyaev R., 2001, MNRAS, 323, 93

Churazov E., Forman W., Jones C., Sunyaev R., Böhringer H., 2004, MNRAS, 347, 29

Costa E., et al., 2008, Space Telescopes and Instrumentation 2008: Ultraviolet to Gamma Ray, eds M.J.L.Turner,K.A.Flanagan, proc. of the SPIE, v. 7011, 70110F

Dolag K., Borgani S., Murante G., Springel V., 2009, MNRAS, 399, 497

Dupke R. A., Arnaud K. A., 2001, ApJ, 548, 141

Elwert G., 1956, ZA, 41, 67

Ezawa H., et al., 2001, PASJ, 53, 595

Forman W., et al., 2007, ApJ, 665, 1057

Gastaldello F., Molendi S., 2004, ApJ, 600, 670

Gilfanov M. R., Sunyaev R. A., Churazov E. M., 1987, SvAL, 13, 3

Hamilton D. R., 1947, ApJ, 106, 457

Hayashi K., Fukazawa Y., Tozuka M., Nishino S., Matsushita K., Takei Y., Arnaud K. A., 2009, PASJ, 61, 1185 
Kaastra J. S., Bleeker J. A. M., Mewe R., 1999, NuPhS, 69, 567

Kahn S. M., et al., 2003, ASPC, 301, 23

Krolik J. H., Raymond J. C., 1988, ApJ, 335, L39

Mathews W. G., Buote D. A., Brighenti F., 2001, ApJ, 550, L31

Mitsuda K., Kelley R., McCammon D., Herder J., Ohashi T., 2008, Journal of Low Temperature Physics, v. 151, 703

Molendi S., Matt G., Antonelli L. A., Fiore F., Fusco-Femiano R., Kaastra J., Maccarone M. C., Perola C., 1998, ApJ, 499, 608

Molnar S. M., Birkinshaw M., Mushotzky R. F., 2006, ApJ, 643, L73

Muleri F., et al., 2009, Journal of Instrumentation, 11, 2

Nicastro F., et al., 2005, Nature, 433, 495

Ostriker J. P., Cen R., 1996, ApJ, 464, 27

Rasmussen A. P., Kahn S. M., Paerels F., Herder J. W. d., Kaastra J., de Vries C., 2007, ApJ, 656,129

Rugge H. R., McKenzie D. L., 1985, ApJ, 297, 338

Sakelliou I., et al., 2002, A\&A, 391, 903

Sanders J. S., Fabian A. C., Allen S. W., Schmidt R. W., 2004, MNRAS, 349, 952

Sanders J. S., Fabian A. C., 2006, MNRAS, 370, 63

Sarazin C. L., 1989, ApJ, 345, 12

Sazonov S. Y., Churazov E. M., Sunyaev R. A., 2002, MNRAS, 333, 191

Sazonov S. Y., Sunyaev R. A., Cramphorn C. K., 2002, A\&A, 393, 793

Shigeyama T., 1998, ApJ, 497, 587

Smith R. K., Brickhouse N. S., Liedahl D. A., Raymond J. C., 2001, ApJ, 556, L91

Soffitta P., et al., 2008, Space Telescopes and Instrumentation 2008: Ultraviolet to Gamma

Ray, eds J.L.Turner, K.A.Flanagan, proc. of the SPIE, v. 7011, 701128

Springel V., Yoshida N., White S. D. M., 2001, NewA, 6, 79

Syunyaev R. A., 1982, SvAL, 8, 175

Werner N., Zhuravleva I., Churazov E., Simionescu A., Allen S. W., Forman W., Jones C., Kaastra J. S., 2009, MNRAS, 398, 23

$\mathrm{Xu} \mathrm{H.,} \mathrm{et} \mathrm{al.,} \mathrm{2002,} \mathrm{ApJ,} \mathrm{579,} 600$

Zhuravleva I. V., Churazov E. M., Sazonov S. Y., Sunyaev R. A., Forman W., Dolag K., 2010a, MNRAS, 403, 129

Zhuravleva I. V., Churazov E. M., Sazonov S. Y., Sunyaev R. A., 2010b, Astronomy Letters, in preparation 